\title{
Neurobehavioral phenotype of autism spectrum disorder associated with germline heterozygous mutations in PTEN
}

Robyn M. Busch ${ }^{1,2}$, Siddharth Srivastava ${ }^{3,4}$, Olivia Hogue ${ }^{5}$, Thomas W. Frazier ${ }^{2,6,7}$, Patricia Klaas ${ }^{1}$, Antonio Hardan ${ }^{8}$, Julian A. Martinez-Agosto ${ }^{9}$, Mustafa Sahin $\mathbb{1}^{3,4}$ and Charis Eng $\mathbb{1}^{2,10,11}$ on behalf of the Developmental Synaptopathies Consortium

\begin{abstract}
Germline mutations in PTEN, the gene that encodes phosphatase and tensin homolog, have been identified in up to $20 \%$ of children with autism spectrum disorder (ASD) and macrocephaly and are associated with marked abnormalities in the white matter of the brain. This study sought to characterize the neurobehavioral phenotype of PTEN-ASD. Comprehensive neurobehavioral evaluations were conducted in 36 participants (ages 3-21 years) with PTEN-ASD and compared to two groups of controls: non-syndromic ASD with macrocephaly (Macro-ASD, $n=25$ ) and those with PTEN mutations without ASD (PTEN-no ASD, $n=23$ ). Linear regression analysis or Kruskal-Wallis tests were used to examine group differences on neurobehavioral measures (cognitive, behavioral, sensory, and adaptive functioning) and, for select measures, one-sample t-tests were used to compare group performance to healthy control norms. These analyses revealed a distinct neuropsychological profile associated with mutations in PTEN suggesting primary disruption of frontal lobe systems (i.e., attention, impulsivity, reaction time, processing speed, and motor coordination). Cognitive deficits in PTEN-ASD are more severe than those in PTEN-no ASD and extend to other areas of neurobehavioral function, specifically, adaptive behavior and sensory deficits. While core ASD symptoms are similar in PTEN-ASD and Macro-ASD, PTEN-ASD had lower clinical ratings of autism severity and showed more sensory abnormalities suggestive of less sensory responsiveness. Together, these results suggest that PTEN-ASD has a distinct neurobehavioral phenotype compared to idiopathic ASD that is likely to warrant special consideration for overall assessment and treatment.
\end{abstract}

\section{Introduction}

Autism spectrum disorder (ASD) is a neurodevelopmental disorder marked by impairments in social communication as well as restricted, repetitive patterns of interests, behaviors, and activities ${ }^{1}$. ASD is highly heritable and may result from a number of genetic anomalies,

\footnotetext{
Correspondence: Charis Eng (engc@ccf.org)

${ }^{1}$ Department of Neurology, Neurological Institute, Cleveland Clinic, Cleveland, $\mathrm{OH}$, USA

${ }^{2}$ Genomic Medicine Institute, Cleveland Clinic, Cleveland, OH, USA Full list of author information is available at the end of the article.

These authors contributed equally: Robyn M. Busch, Siddharth Srivastava Members of the Developmental Synaptopathies Consortium are listed at the end of the paper
}

including chromosomal copy number variation, single gene mutations, epigenetic changes, and complex inheritance $^{2-5}$. Despite this knowledge, only $10-20 \%$ of ASD cases have an identified etiology ${ }^{6}$. As such, most research to date has been conducted on cases with "idiopathic" ASD samples with marked phenotypic and genetic heterogeneity. As a result, it has been difficult to identify therapeutic targets in ASD. One way to surmount this challenge is to study ASD through the lens of specific genetic causes. This approach may serve to distill the complexity and heterogeneity of idiopathic ASD and address the possibility that seemingly unrelated genetic disorders associated with ASD may converge on final

\section{(c) The Author(s) 2019}

(c) (i) Open Access This article is licensed under a Creative Commons Attribution 4.0 International License, which permits use, sharing, adaptation, distribution and reproduction cc) in any medium or format, as long as you give appropriate credit to the original author(s) and the source, provide a link to the Creative Commons license, and indicate if changes were made. The images or other third party material in this article are included in the article's Creative Commons license, unless indicated otherwise in a credit line to the material. If material is not included in the article's Creative Commons license and your intended use is not permitted by statutory regulation or exceeds the permitted use, you will need to obtain permission directly from the copyright holder. To view a copy of this license, visit http://creativecommons.org/licenses/by/4.0/. 
common pathways ${ }^{7,8}$. Therefore, better understanding of mechanistic commonalities among distinct causes may shed light on the pathogenesis of ASD, opening the door to potential targeted treatments ${ }^{9,10}$.

PTEN hamartoma tumor syndrome (PHTS) is one example of a genetic disorder associated with a relatively high prevalence of ASD. PHTS is caused by germline mutations in PTEN, which encodes phosphatase and tensin homolog, resulting in a multitude of presentations that range from increased cancer risks to macrocephaly and neurodevelopmental impairment ${ }^{11-13}$. By some estimates, the prevalence of ASD in PHTS is around 22\% ${ }^{14}$, and studies suggest that up to $20 \%$ of individuals with ASD with macrocephaly may have a pathogenic PTEN variant ${ }^{15-18}$.

Compared to other ASD groups (i.e., macrocephalic ASD, normocephalic ASD) and healthy controls, individuals with ASD and PHTS appear to have a specific pattern of increased and poorly-developed white matter with deficits in processing speed and working memory that extend beyond overall reductions in intellectual functioning ${ }^{19}$. While there is evidence to suggest that patients with PHTS without ASD also have a pattern of cognitive performance that implicates the involvement of frontal lobe systems ${ }^{20}$, these patient groups have never been directly compared across a comprehensive neuropsychological battery to differentiate $P T E N$-specific and ASD-specific deficits. Furthermore, we are unaware of any studies that have comprehensively examined behavioral phenotypes in these patient groups.

The goal of the current study was to comprehensively characterize, cross-sectionally, the neurobehavioral phenotype of PTEN-ASD in a large, prospective cohort and to examine phenotypic differences between these individuals and two groups of controls (i.e., ASD with macrocephaly but without PTEN mutation [Macro-ASD], PTEN mutation without ASD [PTEN-no ASD]). Based on preliminary data in independent samples ${ }^{19,20}$, we hypothesized that: (1) PTEN-ASD would have lower cognitive scores than the other patient groups, particularly on measures of intelligence, processing speed, working memory, and motor functioning; (2) PTEN-ASD would have comparable autism symptom levels to Macro-ASD, but lower intelligence (due to differences in processing speed and/or working memory) and adaptive function; and (3) PTENno ASD would demonstrate reduced performance on measures sensitive to frontal lobe functioning compared to healthy control norms.

\section{Materials and methods Participants}

Participants in this study were recruited from four large tertiary medical centers (Cleveland Clinic, Boston
Children's Hospital, Stanford University Medical Center, and University of California, Los Angeles) as part of an IRB-approved, ongoing, multicenter prospective study designed to examine the natural history of autism and germline heterozygous PTEN mutations (clinicaltrials.gov: NCT02461446). All potential study participants were screened by a clinical psychologist with expertise in ASD to determine if they met DSM-5 diagnostic criteria for ASD. All potential participants also underwent genetic testing to determine the presence/absence of a mutation in PTEN. Individuals were included in the study if they met the following criteria: (1) age 3-21 years; (2) confirmed diagnosis of ASD (based on consensus of expert clinician evaluation and Autism Diagnostic Observation Schedule-2) and/or a confirmed heterozygous mutation in PTEN; (3) English as primary communicative language; and (4) completion of baseline neuropsychological evaluation. In addition, individuals with ASD, but without a PTEN mutation, had to have a occipitofrontal head circumference $\geq 98$ th percentile to be included in the study. Informed consent for study participation was obtained from adult participants and/or a parent or legal guardian. Assent was obtained from all participants age 7 years and older who were cognitively able to provide same.

A total of 84 participants met study inclusion criteria and were categorized into one of the following study groups based on clinical diagnosis and results of PTEN genotyping: PTEN-ASD ( $n=36$; ASD + PTEN mutation), Macro-ASD $(n=25$; ASD with macrocephaly, no PTEN mutation) and PTEN-no ASD $(n=23$; no ASD, PTEN mutation). Sample size for recruitment in the ongoing study was determined based on pilot data which showed effect sizes (Cohen's $d$ ) of 1.5-2.5, when comparing PTEN-ASD and Macro-ASD subjects.

\section{PTEN Genotyping}

Germline genomic DNA was extracted in the Genomic Medicine Institute's Genomic Medicine Biorepository. PCR-based LightCycler mutation scanning and semiautomated PCR-based Sanger sequencing (ABI3730xl in Genomics Core Facility) of exons 1 through 9 and flanking intronic regions of genomic DNA was performed as per routine in the Eng lab since 1997 to reveal germline intragenic mutations in exons 1-9 and in splice sites. All novel variants were checked for presence and frequency in 350 ancestry-matched, sex-matched population controls (standard in Eng lab). PCR-based sequence analysis of the extended promoter region was also performed with Sanger sequencing to reveal promoter variants. All identified promoter variants were subjected to reporter assay as well as routine function interrogation. Multiplex ligationdependent probe amplification was used to reveal large deletions and rearrangements. 


\section{Measures}

All study participants completed a neuropsychological assessment that included cognitive and behavioral measures administered for research purposes. The cognitive battery included age-appropriate measures of global cognitive ability, attention/impulsivity, working memory, processing speed, language, and visuospatial skills. Due to anticipated severe symptom and intellectual impairment in at least a subset of cases, executive functions and motor coordination were not directly assessed, but rather inferred from parent/guardian ratings on standardized questionnaires. Parents/guardians also completed a number of inventories designed to measure autism symptoms, sensory processing, behavioral difficulties, and adaptive functioning. The specific cognitive and behavioral measures used in this study are outlined in Supplementary Table 1. All measures were scored according to published test manuals using age- and/or sex-corrected norms as available/appropriate. Higher scores on the cognitive measures generally reflect better cognitive performance, with the exception of the indices and subscales of the continuous performance test (CPT) and the Behavior Rating Inventory of Executive Function (BRIEF). In contrast, higher scores on the autism, sensory, and behavioral measures are typically indicative of greater symptom severity/behavioral difficulties, with the exception of the Short Sensory Profile (SSP).

\section{Analyses}

Baseline descriptive statistics stratified by diagnosis (PTEN-ASD, Macro-ASD, PTEN-no ASD) were calculated (Table 1). Statistics are presented as means with standard deviations for normally-distributed variables and medians with interquartile ranges for non-normal variables. Not all participants completed all study measures. Incomplete evaluations were largely due to the patient's inability to tolerate testing or to time constraints. For those patients who were assessed, but who could not complete a particular measure due to low functional capacity, a score one point lower than the lowest possible score for that measure was assigned for purposes of data analyses. Assessments that were incomplete due to time constraints were considered missing at random.

Chi-square analyses were used to examine differences in the proportion of missense versus truncating mutation types between PTEN-ASD and PTEN-no ASD.

The study groups were compared on cognitive and behavioral measures using the PTEN-ASD group as a reference. For normally-distributed variables, linear regression analysis was used. Residuals by fitted value plots were examined to verify homoscedasticity. Kruskal-Wallis tests with the Dwass, Steel, CritchlowFligner pairwise comparison tests were used to compare the non-normal variables. Due to the significant age
Table 1 Demographic characteristics of study groups

\begin{tabular}{|c|c|c|c|}
\hline & $\begin{array}{l}\text { PTEN-ASD } \\
n=36\end{array}$ & $\begin{array}{l}\text { Macro-ASD } \\
n=25\end{array}$ & $\begin{array}{l}\text { PTEN-no ASD } \\
n=23\end{array}$ \\
\hline \multirow[t]{3}{*}{$A g e^{a}$} & $8.8(5.1)$ & $12.9(4.9)$ & $8.6(4.1)$ \\
\hline & Range & Range & Range \\
\hline & $3.1-19.6$ & $4.2-21.5$ & $3.6-18.7$ \\
\hline \multicolumn{4}{|l|}{ Sex } \\
\hline Female & $8(22.2)$ & $3(12.0)$ & $9(39.1)$ \\
\hline Male & $28(77.8)$ & $22(88.0)$ & $14(60.9)$ \\
\hline \multicolumn{4}{|l|}{ Race } \\
\hline White/Caucasian & $29(80.6)$ & $14(56.0)$ & $17(73.9)$ \\
\hline Black/African American & $1(2.8)$ & $1(4.0)$ & 0 \\
\hline Asian & 0 & $5(20.0)$ & $2(8.7)$ \\
\hline Multiracial & $4(11.1)$ & $5(20.0)$ & $3(13.0)$ \\
\hline $\begin{array}{l}\text { Unknown/Not } \\
\text { Reported }\end{array}$ & $2(5.6)$ & 0 & $1(4.4)$ \\
\hline \multicolumn{4}{|l|}{ Ethnicity } \\
\hline Hispanic & $6(16.6)$ & $3(12.0)$ & $1(4.4)$ \\
\hline Not Hispanic & $29(80.6)$ & $22(88.0)$ & $21(91.3)$ \\
\hline $\begin{array}{l}\text { Unknown/Not } \\
\text { reported }\end{array}$ & $1(2.9)$ & 0 & $1(4.4)$ \\
\hline
\end{tabular}

ASD $=$ autism spectrum disorder

a Values presented as mean (standard deviation). All other values in table are presented as number (percentage)

difference among groups, the effect of age on each cognitive and behavioral measure was tested using Pearson or Spearman's correlations where appropriate. If age was significantly associated with the measure, it was included as a control variable. Exploratory multiple regression analyses also examined IQ as a function of both group and measures of frontal lobe functioning, including processing speed and working memory.

The Autism Diagnostic Observation Schedule-Second Edition (ADOS-2) was administered only to participants with ASD after diagnosis was confirmed by an expert clinical psychologist. PTEN-ASD and Macro-ASD were compared using the Wilcoxon rank-sum test.

One sample $t$-tests were used to compare mean performance of PTEN-no ASD to healthy control norms.

Given the exploratory nature of this study and relatively small sample sizes, we report effect sizes (Cohen's $d$ ) in addition to $p$-values for all analyses rather than correcting for multiple comparisons. Effect sizes were interpreted as follows: small effect $=0.20$, medium effect $=0.50$, large effect $=0.80$. Groups displayed homogeneity of variance for all comparisons. Analyses were two-sided and conducted using SAS Studio v. 3.3. SAS code is available upon request. 


\section{Results}

\section{Demographic characteristics}

There were no significant differences in sex, race, or ethnicity among the three study groups. Macro-ASD was significantly older on average than both PTEN-ASD and PTEN-no ASD $(p=0.002)$ [Table 1].

\section{Genetic analyses}

A summary of the germline PTEN variants observed in the study cohort is provided in Supplementary Table 2. There was a similar proportion of missense mutations $(52.8 \%$ vs. $34.8 \%, p=0.176)$ and truncating variants ( $44.4 \%$ vs. $60.9 \%, p=0.218)$ in PTEN-ASD compared to PTEN-no ASD.

\section{Neurobehavioral profile of PTEN-ASD}

PTEN-ASD demonstrated poorer performance than $P T E N$-no ASD in every cognitive domain assessed, with effect sizes for most measures in the medium to large range (i.e., Cohen's $d$ range 0.41-2.21). Caregivers of children in the PTEN-ASD group also observed greater symptoms of behavioral and sensory dysfunction than caregivers of children in the PTEN-no ASD group, with similar range effects ( $d$ range $0.49-2.06$ ) (Supplementary Table 3 and Fig. 1).

When compared to Macro-ASD, children with PTENASD demonstrated greater cognitive impairments on measures of nonverbal intelligence $(d=0.65)$, attention $(d=0.73)$, reaction time $(d=0.85)$, and motor coordination $(d=0.69)$. While the threshold for statistical significance was not reached, medium to large effect sizes were also observed on measures of impulsivity $(d=0.61)$ and processing speed $(d=0.50)$ (Supplementary Table 4 and Fig. 1).

When controlling for working memory, the difference in FSIQ between PTEN-ASD and PTEN-no ASD decreased from 41.9 points to 6.3 points and the difference in FSIQ between PTEN-ASD and Macro-ASD decreased from 11.4 points to 5.5 points. Among subjects who completed the subtests that go into the Weschler Processing Speed Index, controlling for processing speed decreased the difference in FSIQ between PTEN-ASD and PTEN-no ASD from 46.7 points to 18.4 points and decreased the difference in FSIQ between PTEN-ASD and Macro-ASD from 19.3 points to 12.1 points.

PTEN-ASD had lower ADOS-2 Calibrated Severity Scores than Macro-ASD indicating less severe autism symptoms in this genetic sub-group $(d=0.56)$. While parents of both ASD groups noted less social responsiveness and more repetitive behavior than the PTEN-no ASD group, the two ASD groups did not significantly differ from each other on these scales (i.e., SRS and RBSR) (Supplementary Tables 3 and 4). On caregiver-report questionnaires, PTEN-ASD were noted to have greater difficulties with sensory functioning than Macro-ASD $(d=0.62)$, particularly on the under-responsive/seeks sensation $(d=0.50)$, low energy/weak $(d=0.49)$, and taste/smell sensitivity $(d=0.50)$ scales (Supplementary Table 4 and Fig. 2). The two groups had similar symptoms on behavioral and adaptive behavior measures. (Supplementary Table 4).

Group performance on all cognitive and behavioral measures is summarized in Supplementary Tables 3 and

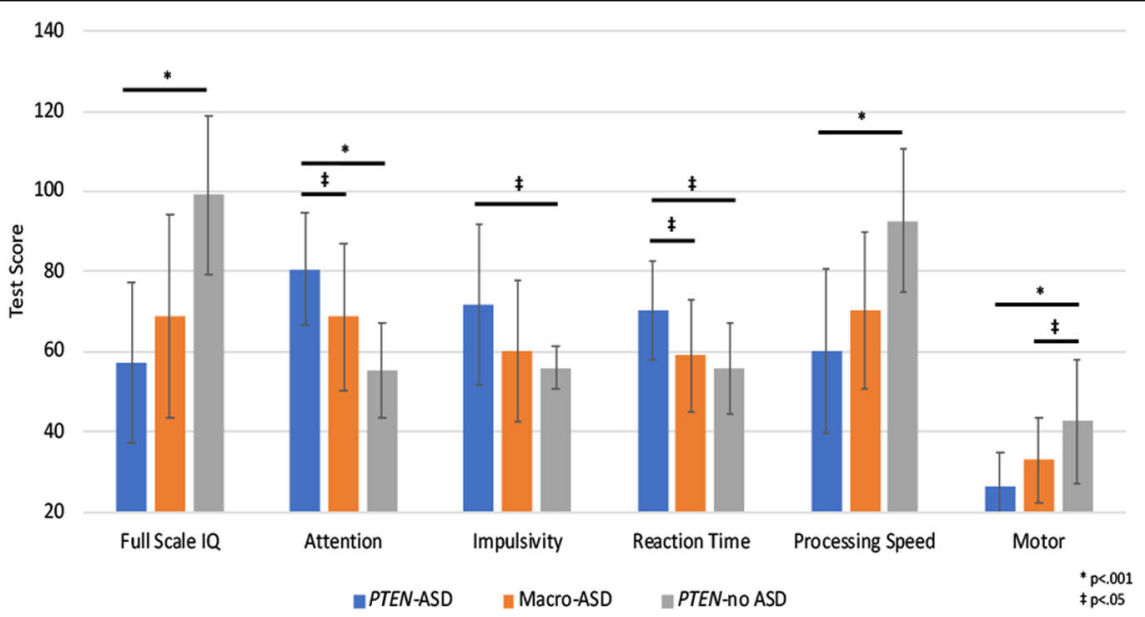

Fig. 1 Group differences in cognitive and motor functioning. Higher scores indicate better cognitive performance for all domains, except attention, impulsivity, and reaction time. Full Scale IQ and processing speed are reported on a standard score scale $(M=100, S D=15)$, attention, impulsivity, and reaction time are reported on a $t$-score scale $(M=50, S D=10)$, and motor is reported as a raw score. The measures used to assess the noted cognitive domains are outlined in Supplementary Table 1. ASD, autism spectrum disorder 


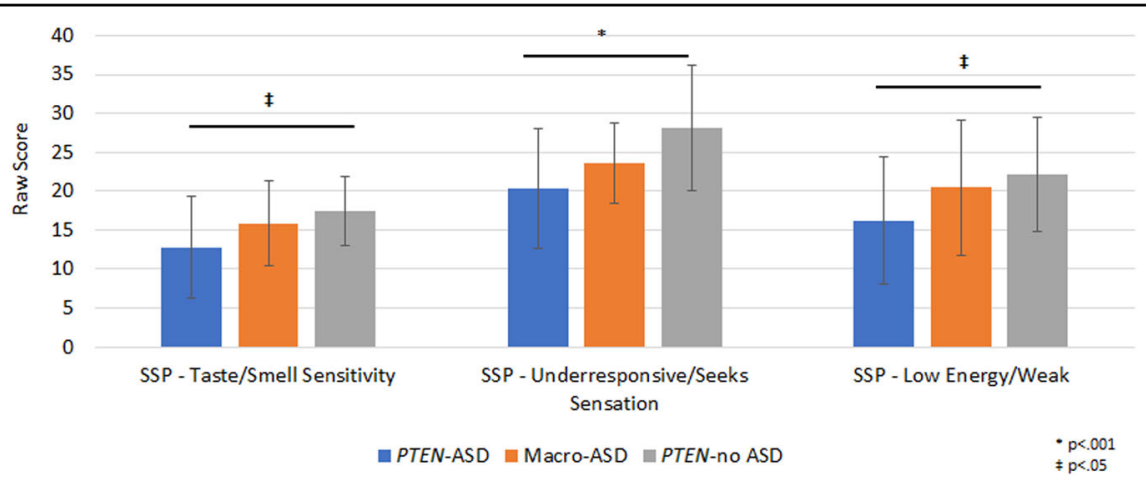

Fig. 2 Group differences in sensory functioning. Higher scores indicate fewer sensory difficulties. Typical performance score ranges: Tactile Sensitivity 30-35, taste/smell sensitivity 15-20, under responsive/seeks sensation 27-35, low energy/weak 26-30. SSP, short sensory profile, ASD, autism spectrum disorder

Table 2 Summary of mean group performance on neurobehavioral measures ${ }^{a}$ by domain

\begin{tabular}{llll}
\hline & PTEN-ASD & Macro-ASD & PTEN-no ASD \\
\hline Verbal intelligence & Severely impaired & Moderate impairment & High average \\
Nonverbal intelligence & Severely impaired & Mildly impaired & Average \\
Attention & Severely impaired & Mildly impaired & Average \\
Working memory & Moderately impaired & Moderately impaired & Low average to average \\
Impulsivity & Mildly impaired & Low average & Average \\
Processing speed & Moderately impaired & Mildly imapired & Average \\
Reaction time & Mildly impaired & Low average & Average \\
Executive functions & Mildly impaired & Mildly impaired & Low average \\
Expressive language & Moderately impaired & Mildly impaired & Average \\
Receptive language & Moderately impaired & Mildly impaired & High average \\
Visuospatial & Moderately impaired & Mildly impaired & Average \\
Motor & Severely impaired & Moderately impaired & Mildly impaired \\
Sensory functioning & Severely impaired & Severely impaired & Moderately impaired \\
Problem behavior & Moderately impaired & & Moderately impaired \\
\hline ASD a & & Average
\end{tabular}

ASD autism spectrum disorder

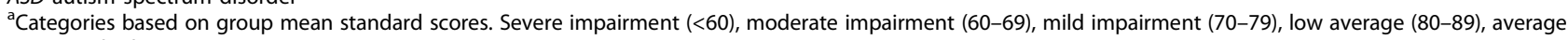
(90-109), high average (110-119)

4, respectively, and a summary of observed neurobehavioral impairments by domain is provided in Table 2. It was necessary to control for age on CPT Commissions only.

\section{Neurobehavioral profile of PTEN-no ASD}

$P T E N$-no ASD did not significantly differ from established healthy control norms on measures of global cognitive ability. However, they had reduced mean scores compared to healthy controls on measures of subjective working memory $(p=0.011, d=0.81)$, impulsivity $(p=0.002, d=0.75)$, visuomotor integration $(p=0.012$, $d=0.62)$, and motor coordination $(p<0.001, d=1.48)$. In contrast, their scores on a measure of receptive vocabulary were significantly higher than established healthy controls norms $(p=0.021, d=0.60)$ (Fig. 3).

On adaptive functioning measurements, $P T E N$-no ASD had reduced scores on the Motor Skills $(p<0.001, d=$ $0.95)$ and Internalizing $(p=0.035, d=0.57)$ subscales of the Vineland Adaptive Behavior Inventory-Second Edition compared to healthy control norms. Caregivers of children in the PTEN-no ASD group also reported more repetitive behaviors on the Repetitive Behavior Scale compared to healthy control norms $(p=0.004, d=0.99)$. 


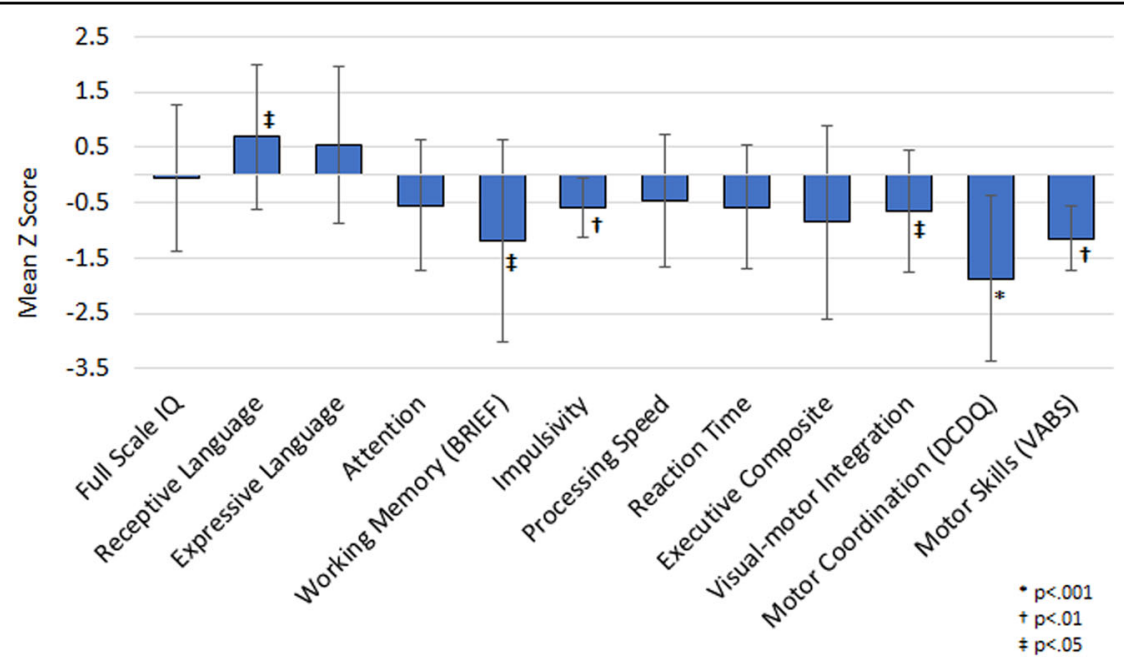

PTEN-no ASD

Fig. 3 Cognitive performance in PTEN-ASD compared to healthy control norms. Upgoing bars indicate performances higher than normative mean $z$-score of 0 , and downgoing bars indicate performances lower than normative mean z-score of 0 . BRIEF, Behavior Rating Inventory of Executive Function, DCDQ, Developmental Coordination Disorder Questionnaire, VABS, Vineland Adaptive Behavior Scale, ASD, autism spectrum disorder

Interestingly, the mean scores of PTEN-no ASD were below normative expectation on the total score of the Short Sensory Profile suggesting greater sensory difficulties. Examination of the subtest scores revealed the most notable difficulties with low energy/weakness and auditory filtering.

\section{Discussion}

We have identified a distinct neuropsychological profile associated with mutations in PTEN suggesting primary disruption of frontal lobe systems ${ }^{21,22}$. Specifically, individuals with PTEN mutations showed reduced performance on measures of attention, impulsivity, reaction time, processing speed, and motor coordination, consistent with our pilot findings in independent patient cohorts $^{19,20}$. This pattern of cognitive deficits may be related to neuroanatomical abnormalities. PTEN is a dualspecificity phosphatase that is a major inhibitor of the PI3K-AKT pathway ${ }^{23-25}$ with downstream effects on mTOR signaling, which helps regulate neural cell growth and proliferation ${ }^{26}$. Germline mutations in PTEN are associated with macrocephaly, with brain MRI showing markedly larger brain volumes as well as increased white matter hypointensities compared to other patient groups and healthy controls ${ }^{19,27,28}$. Even after controlling for total intracranial volume, volumetric differences remain between individuals with PTEN mutations and healthy controls in total white matter as well as in a number of specific brain regions, including the corpus callosum and cerebellum. While cortical thickness appears to vary across brain regions, our recent findings suggest reduced cortical thickness in the frontal regions of individuals with
PTEN mutations ${ }^{19,28}$, which may account for our cognitive observations.

Pten $^{\text {m3m4 }}$ murine model, a constitutional Pten knock-in model with autism-like phenotypes, demonstrate upregulated expression of genes implicated in myelination, including myelin basic protein and myelin proteolipid protein, possibly contributing to increased volumes of white matter structures ${ }^{19}$. Oligodendrocyte development relies on precise transcriptional control of genes involved in structural support of myelin ${ }^{29}$, and deregulation of this tightly controlled process may lead to white matter abnormalities. While behavioral studies in $\operatorname{Pten}^{m 3 m 4}$ mice have focused primarily on autism phenotypes (e.g., social interaction, repetitive behavior, anxiety ${ }^{16}$, these mice do not appear to have global cognitive dysfunction and there is some evidence to suggest motor coordination deficits and social difficulties as observed in humans with PTEN mutations ${ }^{16}$. To our knowledge, attention, working memory and other more "frontal" type tasks have not yet been assessed in rodent models of Pten. Further research in this regard will be needed to validate our findings. It is also important to note that we cannot rule out the possibility that cerebellar dysfunction may contribute to this pattern given the known role of the cerebellum in many aspects of motor and cognitive functioning ${ }^{30-32}$.

While both PTEN groups showed cognitive patterns suggesting primary involvement of frontal lobe systems, PTEN-ASD had deficits that were more severe than $P T E N$-no ASD and extended to other areas of neurobehavioral function. Specifically, cognitive deficits in PTENno ASD tended to be mild and largely restricted to measures sensitive to frontal lobe function, whereas 
PTEN-ASD showed frontal deficits in the moderate to severe range accompanied by moderate to severe impairments in intellectual functioning and moderate deficits in both expressive and receptive language suggesting involvement of more widespread brain regions (Table 2). Interestingly, both PTEN groups reported deficits in sensory functioning, with the most notable problems on the under-responsive/seeks sensation, low energy/weak and taste/smell sensitivity subscales of the Short Sensory Profile (Fig. 3 and Supplementary Tables 3 and 4). The more severe neurobehavioral phenotype observed in PTEN-ASD may be related to more extensive brain abnormalities compared to PTEN-no ASD. While we are unaware of any studies that have directly compared neuroimaging characteristics of these two patient groups, anectodally PTEN-ASD appears to have greater white matter volume than PTEN-no ASD. This hypothesis remains to be confirmed in future studies with agematched samples.

Similarly, while both ASD groups demonstrated rather global cognitive impairments, PTEN-ASD showed more severe impairments than those with Macro-ASD, particularly on measures associated with frontal systems dysfunction (e.g., attention, reaction time, motor function) (Supplementary Table 4). These findings are consistent with prior research in a small independent cohort suggesting reduced cognitive functioning in PTEN-ASD versus idiopathic $\mathrm{ASD}^{19}$ and likely reflect specific brain alterations including white matter abnormalities associated with PTEN mutations as previously discussed. In contrast, core ASD symptoms in PTEN-ASD appear to be similar to those seen in idiopathic ASD. PTEN-ASD and Macro-ASD reported similarities in social responsiveness and repetitive behavior (as assessed by the SRS and RBS, respectively); however, PTEN-ASD had lower clinical ratings of autism severity on the ADOS-2. This may reflect the passive/slower presentation of PTEN-ASD rather than a true reduction in autism severity. Indeed, caregiver responses on a sensory functioning measure suggest that PTEN-ASD often presents with low energy/ weakness and under responsiveness. These findings are quite consistent with parent reports as well as our qualitative observations in the clinic.

Importantly, these study results suggest that the slow processing speed/reaction times, attention/workingmemory difficulties, and motor deficits observed in individuals with PTEN mutations likely impact other aspects of their test performance. While mean FSIQ in PTEN-no ASD was within the average range (i.e., standard score of 99.2), scores on an untimed measure of receptive language (Peabody Picture Vocabulary Test) known to be highly correlated with FSIQ were substantially higher (i.e., standard score 110.4, high average range). This suggests that FSIQ, which has attention/working memory, speed, and motor components, may underestimate general cognitive ability in PTEN patients. Indeed, our data would support this conclusion as differences in FSIQ observed between groups were much less apparent after adjusting for working memory and processing speed.

The rather consistent pattern of neurobehavioral deficits observed in individuals with PTEN muations is likely to have important clinical and educational implications, particularly for those diagnosed in childhood, and help contribute to our understanding of the underlying pathophysiology of autism symptoms and/or cognitive deficits in those with PTEN mutations. As such, comprehensive neuropsychological evaluation should be considered in children, adolescents, and young adults with PTEN mutations to thoroughly assess social and behavioral functioning as well as to identify cognitive strengths and weaknesses. Clinicians, and researchers, should also be sure to assess attention, speed and motor functions as part of any cognitive evaluation and to take those results into account when interpreting other test data and making clinical and educational recommendations. These type of assessments can inform tailored recommendations for academic accommodations and targeted interventions to optimize cognitive performance, adaptive functioning, and educational outcomes. For example, individuals with slow processing speed and/or attention/working memory difficulties may require information to be presented at a more deliberate pace and may benefit from brief delays in conversation to permit the formulation of their response. These individuals may also require academic accommodations to permit more time for assignments and tests, and educational expectations should be tempered. Tests that rely on motor coordination and speed should be avoided given the marked motor deficits associated with mutations in PTEN.

Taken together, results of this study suggest that children with PTEN-ASD have a distinct neurobehavioral phenotype and, as such, are likely to vary in several aspects of clinical presentation from patients with idiopathic ASD. Clinicians should make note of these cognitive and behavioral differences and be wary not to underidentify autism in individuals with PTEN mutations, as clinical observations may confound a relatively passive/ slower processing phenotype with autism symptom severity. As in all individuals with autism, early identification is key to accessing evidence-based early interventions and maximizing developmental trajectories ${ }^{33-35}$. Comprehensive neuropsychological evaluation is recommended for the identification of cognitive strengths as well as weaknesses that may require remediation and/or accommodation. Similarly, the marked deficits in motor and sensory functioning that often accompany PTENASD are likely to warrant thorough occupational and physical therapy evaluations for most patients. In contrast 
to idiopathic ASD, our results suggest that individuals with PTEN-ASD may require less in terms of assessment and/or intervention planning for challenging behavior and more with regard to cognitive rehabilitation/treatment. Ultimately, it is important for clinicians, parents, caregivers, and teachers to be made aware of these important phenotypic differences in order to obtain appropriate interventions and maximize day-to-day functioning.

There are a few limitations of the current study that deserve mention. There was a small subset of children in the study who were too low functioning to complete all cognitive measures. Rather than limit our dataset only to those with complete data and risk the potential of overestimating cognitive ability in this cohort, we assigned one lower than the lowest possible score for tasks that were attempted, but could not be completed. Further, while sample sizes in this cohort are among the highest reported to date, analyses for some measures were underpowered to detect medium effect sizes. In order to address this issue, we calculated effect sizes for each of the comparisons rather than rely solely on traditional $p$-values. It is also important to note that the Macro-ASD group was older than the two PTEN groups and, while not statistically significant, had a smaller proportion of females. Expanded recruitment in the overarching study is planned to achieve better demographic balance among groups and increase sample size, thereby improving power to detect smaller effects. Finally, while these cross-sectional data highlight some distinct neurobehavioral characteristics associated with PTEN-ASD, they give only a snapshot of functioning at a single time point in childhood/adolescence. Thus, efforts are currently underway to follow these children longitudinally and to extend recruitment to a broader age range (18 months to 45 years) in order to examine the evolution of neurobehavioral functioning in PTEN-ASD throughout development and into adulthood. Finally, future research will seek to better understand the mechanisms that drive the neurobehavioral differences we have observed. Our future work will examine relationships between neurobehavioral variables and underlying molecular and neurophysiological systems as well as neuroimaging measures. Equipped with this information, we hope to build a comprehensive longitudinal cross-level model that builds key links from PTEN mutations to neurobehavioral outcomes.

\footnotetext{
Acknowledgements

We are sincerely indebted to the generosity of the families and patients in PTEN clinics across the United States who contributed their time and effort to this study. We would also like to thank the PTEN Hamartoma Syndrome Foundation and the PTEN Research Foundation for their continued support in PTEN research. This study was funded, in part, by the National Institute of Health Developmental Synaptopathies Consortium (U54NS092090; PI/Network Director: Sahin; Project 2 Leaders/PIs: Eng \& Frazier) and the Ambrose Monell Foundation (to CE). The Developmental Synaptopathies Consortium (U54NS092090) is part of the Rare Diseases Clinical Research Network (RDCRN),
}

an initiative of the Office of Rare Diseases Research (ORDR), National Center for Advancing Translational Sciences (NCATS). Research reported in this publication was supported by the National Institute of Neurological Disorders and Stroke of the National Institutes of Health (NINDS), Eunice Kennedy Shriver National Institute of Child Health \& Human Development (NICHD), National Institute of Mental Health (NIMH) and National Center for Advancing Translational Sciences (NCATS). The content is solely the responsibility of the authors and does not necessarily represent the official views of the National Institutes of Health (NIH). CE is the Sondra J. and Stephen R. Hardis Endowed Chair of Cancer Genomic Medicine at the Cleveland Clinic and an ACS Clinical Research Professor. MS is the Rosamund Stone Zander Chair at Boston Children's Hospital.

\section{Author details}

'Department of Neurology, Neurological Institute, Cleveland Clinic, Cleveland, $\mathrm{OH}$, USA. ${ }^{2}$ Genomic Medicine Institute, Cleveland Clinic, Cleveland, OH, USA. ${ }^{3}$ Department of Neurology, Boston Children's Hospital, Harvard Medical School, Boston, MA, USA. ${ }^{4}$ Harvard Medical School and Boston Children's Hospital, Boston, MA, USA. ${ }^{5}$ Department of Quantitative Health Sciences, Cleveland Clinic, Cleveland, OH, USA. ${ }^{6}$ Autism Speaks, Cleveland, OH, USA. ${ }^{7}$ Pediatrics Institute, Cleveland Clinic, Cleveland, OH, USA. ${ }^{8}$ Psychiatry and Behavioral Sciences, Stanford University Medical Center, Stanford, CA, USA. ${ }^{9}$ Department of Human Genetics, University of California Los Angeles, Los Angeles, CA, USA. ${ }^{10}$ Taussig Cancer Institute, Cleveland Clinic, Cleveland, $\mathrm{OH}$, USA. "'Department of Genetics and Genome Sciences, Case Western Reserve University, Cleveland, OH, USA

\section{Conflict of interest}

Dr. Busch has no relevant financial interests or potential conflicts of interest to report. Dr. Srivastava has no relevant financial interests or potential conflicts of interest to report. Ms. Hogue has no relevant financial interests or potential conflicts of interest to report. Dr. Frazier has received federal funding or research support from, acted as a consultant to, received travel support from, and/or received a speaker's honorarium from the Cole Family Research Fund, Simons Foundation, Ingalls Foundation, Forest Laboratories, Ecoeos, IntegraGen, Kugona LLC, Shire Development, Bristol-Myers Squibb, Roche Pharma, National Institutes of Health, and the Brain and Behavior Research Foundation. Dr. Klaas has no relevant financial interests or potential conflicts of interest to report. Dr. Hardan has no relevant financial interests or potential conflicts of interest to report. Dr. Martinez-Agosto has no relevant financial interests or potential conflicts of interest to report. Dr. Sahin reports grant support from Novartis, Roche, Pfizer, Ipsen, LAM Therapeutics and Quadrant Biosciences, and he has served on Scientific Advisory Boards for Sage, Roche Celgene and Takeda, all unrelated to this project. Dr. Eng has no relevant financial interests or potential conflicts of interest to report.

Supplementary Information accompanies this paper at (https://doi.org/ 10.1038/s41398-019-0588-1).

Received: 17 May 2019 Revised: 4 September 2019 Accepted: 16 September 2019

Published online: 08 October 2019

\section{References}

1. American Psychiatric Association. Diagnostic and Statistical Manual of Mental Disorders, Fifth Edition. American Psychiatric Association. Retrieved July 5, 2016, from http://psychiatryonline.org/doi/book/10.1176/appi. books.9780890425596. (2013).

2. de la Torre-Ubieta, L., Won, H., Stein, J. L. \& Geschwind, D. H. Advancing the understanding of autism disease mechanisms through genetics. Nat. Med. 22, 345-361 (2016).

3. Abrahams, B. S. \& Geschwind, D. H. Advances in autism genetics: on the threshold of a new neurobiology. Nat. Rev. Genet. 9, 341-355 (2008).

4. Loke, Y. J., Hannan, A. J. \& Craig, J. M. The role of epigenetic change in autism spectrum disorders. Front Neurol. 6, 107 (2015).

5. Kiser, D. P., Rivero, O. \& Lesch, K.-P. Annual research review: the (epi) genetics of neurodevelopmental disorders in the era of whole-genome sequencing-unveiling the dark matter. J. Child Psychol. Psychiatry 56, 278-295 (2015). 
6. Geschwind, D. H. Genetics of autism spectrum disorders. Trends Cogn. Sci. 15, 409-416 (2011).

7. Geschwind, D. H. Autism: many genes, common pathways? Cell 135, 391-395 (2008).

8. Tilot, A. K. et al. Neural transcriptome of constitutional Pten dysfunction in mice and its relevance to human idiopathic autism spectrum disorder. Mol. Psychiatry 21, 118-125 (2016).

9. Plummer J. T., Gordon A. J. \& Levitt P. The genetic intersection of neurodevelopmental disorders and shared medical comorbidities-Relations that translate from bench to bedside. Front Psychiatry. 7. https://doi.org/10.3389/ fpsyt.2016.00142 (2016).

10. Sahin M. \& Sur M. Genes, circuits, and precision therapies for autism and related neurodevelopmental disorders. Science. 350. https://doi.org/10.1126/ science.aab3897 (2015).

11. Eng C. (1993): PTEN Hamartoma Tumor Syndrome. In: Pagon R. A., et al., editors. GeneReviews $\left({ }^{\circledR}\right)$. Seattle (WA): University of Washington, Seattle. Retrieved March 27, 2017, from http://www.ncbi.nlm.nih.gov/books/NBK1488/.

12. Ngeow, J., Sesock, K. \& Eng, C. Clinical implications for germline PTEN spectrum disorders. Endocrinol. Metab. Clin. North Am. 46, 503-517 (2017).

13. Yehia, L. \& Eng, C. 65 years of the double helix: one gene, many endocrine and metabolic syndromes: PTEN-opathies and precision medicine. Endocr. Relat. Cancer 25, T121-T140 (2018).

14. Tan, M.-H. et al. A clinical scoring system for selection of patients for PTEN mutation testing is proposed on the basis of a prospective study of 3042 probands. Am. J. Hum. Genet. 88, 42-56 (2011).

15. Butler, M. G. et al. Subset of individuals with autism spectrum disorders and extreme macrocephaly associated with germline PTEN tumour suppressor gene mutations. J. Med Genet. 42, 318-321 (2005).

16. Tilot, A. K., Frazier, T. W. \& Eng, C. Balancing proliferation and connectivity in PTEN-associated autism spectrum disorder. Neurotherapeutics 12, 609-619 (2015).

17. Lainhart, J. E. et al. Head circumference and height in autism: a study by the collaborative program of excellence in autism. Am. J. Med Genet A. 140, 2257-2274 (2006).

18. Varga, E. A. et al. The prevalence of PTEN mutations in a clinical pediatric cohort with autism spectrum disorders, developmental delay, and macrocephaly. Genet Med. 11, 111-117 (2009).

19. Frazier, T. W. et al. Molecular and phenotypic abnormalities in individuals with germline heterozygous PTEN mutations and autism. Mol. Psychiatry 20 1132-1138 (2015).

20. Busch, R. M. et al. The cognitive characteristics of PTEN Hamartoma tumor syndromes. Genet Med. 15, 548-553 (2013).
21. Chayer, C. \& Freedman, M. Frontal lobe functions. Curr. Neurol. Neurosci. Rep. 1, 547-552 (2001).

22. Stuss D. T. et al. Fractionation and Localization of Distinct Frontal Lobe Processes: Evidence from Focal Lesions in Humans. Oxford University Press. Retrieved July 16, 2019, from https://www.oxfordscholarship.com/view/10.1093/acprof:oso/ 9780195134971.001.0001/acprof-9780195134971-chapter-25. (2002).

23. Georgescu, M.-M. PTEN Tumor suppressor network in PI3K-Akt pathway control. Genes Cancer 1, 1170-1177 (2010).

24. Myers, M. P. et al. P-TEN, the tumor suppressor from human chromosome 10q23, is a dual-specificity phosphatase. Proc. Natl Acad. Sci. USA 94, 9052-9057 (1997)

25. Stambolic, V. et al. Negative regulation of PKB/Akt-dependent cell survival by the tumor suppressor PTEN. Cell 95, 29-39 (1998).

26. Takei N. \& Nawa H. mTOR signaling and its roles in normal and abnormal brain development. Front Mol Neurosci. 7. https://doi.org/10.3389/ fnmol.2014.00028 (2014).

27. Vanderver, A. et al. Characteristic brain magnetic resonance imaging pattern in patients with macrocephaly and PTEN mutations. Am. J. Med Genet A. 164A, 627-633 (2014).

28. Busch, R. M. et al. Structural imaging findings in individuals with PTEN Hamartoma Tumor Syndrome (PHTS) and cognitive dysfunction. (Presented at the Society for Neuroscience, San Diego, CA, 2018).

29. Lee P. R. \& Fields R. D. (2009): Regulation of myelin genes implicated in psychiatric disorders by functional activity in axons. Front Neuroanat. 3, https:// doi.org/10.3389/neuro.05.004.2009 (2009).

30. O'Halloran, C. J., Kinsella, G. J. \& Storey, E. The cerebellum and neuropsychological functioning: a critical review. J. Clin. Exp. Neuropsychol. 34, 35-56 (2012).

31. Stoodley, C. J. The cerebellum and cognition: evidence from functional imaging studies. Cerebellum 11, 352-365 (2012).

32. Sundberg, M. \& Sahin, M. Cerebellar development and autism spectrum disorder in tuberous sclerosis complex. J. Child Neurol. 30, 1954-1962 (2015).

33. Green, J. et al. Randomised trial of a parent-mediated intervention for infants at high risk for autism: longitudinal outcomes to age 3 years. J. Child Psychol. Psychiatry 58, 1330-1340 (2017).

34. Almirall, D. et al. Longitudinal effects of adaptive interventions with a speechgenerating device in minimally verbal children with ASD. J. Clin. Child Adolesc. Psychol. 45, 442-456 (2016).

35. Rogers, S. J. et al. Autism treatment in the first year of life: a pilot study of infant start, a parent-implemented intervention for symptomatic infants. J. Autism Dev. Disord. 44, 2981-2995 (2014).

\section{on behalf of the Developmental Synaptopathies Consortium}

Simon K. Warfield ${ }^{12}$, Benoit Scherrer ${ }^{12}$, Kira Dies ${ }^{13}$, Rajna Filip-Dhima ${ }^{13}$, Amanda Gulsrud ${ }^{14}$, Ellen Hanson ${ }^{15}$ \& Jennifer M. Phillips ${ }^{16}$

${ }^{12}$ Department of Radiology, Boston Children's Hospital, Boston, MA, USA. ${ }^{13}$ Department of Neurology, Boston Children's Hospital, Boston, MA, USA. ${ }^{14}$ UCLA Semel Institute for Neuroscience \& Human Behavior, David Geffen School of Medicine at UCLA, Los Angeles, CA, USA. ${ }^{15}$ Department of Developmental Medicine, Boston Children's Hospital, Boston, MA, USA. ${ }^{16}$ Department of Psychiatry, Stanford University, Stanford, CA, USA 